\title{
Physicochemical Characterization of Simulated Intestinal Fed-State Fluids Containing Lyso-Phosphatidylcholine and Cholesterol
}

\author{
D. G. Fatouros ${ }^{1,2}$, I. Walrand ${ }^{1}$, B. Bergenstahl ${ }^{3}$, and A. Mullertz ${ }^{4}$ \\ 'Department of Pharmaceutics and Analytical Chemistry Faculty of Pharmaceutical Sciences, \\ University of Copenhagen, Denmark \\ ${ }^{2}$ Current address: School of Pharmacy and Biomedical Sciences, University of Portsmouth, \\ Portsmouth, Hampshire, PO1 2DT, UK \\ ${ }^{3}$ Department of Food Technology, Center for Chemistry and Chemical Engineering, \\ Lund University, Lund, Sweden \\ ${ }^{4}$ Bioneer:FARMA, Faculty of Pharmaceutical Sciences, University of Copenhagen, Denmark
}

\begin{abstract}
An array of simulated intestinal fed-state fluids, where phosphatidylcholine (PC) was replaced by lysophosphatidylcholine (LPC) and cholesterol (Chol) was added, were visualized with Cryogenic Transmission Electron Microscopy (Cryo-TEM). Micelles were the dominating structural features, emphasizing the micellar-forming characteristics of LPC. Upon increase of the monoglyceride (MG) level, unilamellar vesicles and multivesicular structures were formed. These findings suggest the solubilization of poorly soluble drugs might be affected by the intermediate colloidal phases produced in the gastrointestinal tract.
\end{abstract}

\section{INTRODUCTION}

S mall intestinal fluid contains various endogenous surfactants, including bile salts (BS) and phospholipids (PL), which form mixed micelles with high solubilizing capacity for many poorly soluble drugs $(1,2)$. In general, mean fasted-state BS concentrations typically range from 1.5 to $6 \mathrm{mM}(3,4)$, while mean postprandial concentrations typically range from 5 to 25 $\mathrm{mM}$ depended on the composition of the meal $(5,6)$.

Knowledge of the composition and characteristics of human intestinal fluid is expanding; however, in order for this information to be translated into useful predictive dissolution media, more studies including physicochemical characterization of media simulating the human intestinal fluids are needed $(7,8)$. The intermediate phases produced during lipid digestion can play a significant role in drug solubilization and trafficking in the gastrointestinal tract, thereby influencing the overall performance of the formulation $(9,10)$. In light of this, characterization of such media can offer important information on the role of intermediate phases of lipid digestion and drug solubilization in the gastrointestinal tract.

Fed-state simulated intestinal fluids are often composed of sodium taurocholate, phosphatidylcholine, monoolein, and oleic acid, with the latter two compounds in a ratio of $1: 2$. However, recent studies have shown that the actual ratio present in human intestinal fluids is more likely to be 6:1 rather than 2:1 (6). Moreover, cholesterol and LPC are

${ }^{2}$ Corresponding author. also present. Given that the apparent solubility of poorly water-soluble drugs is related to the colloidal phases produced in the small intestine, studies characterizing these media offer insights into the mechanisms underlying the digestion processes. During lipid digestion, the phospholipids are hydrolyzed to lysophospholipids and free fatty acids. In an attempt to elucidate the impact of LPC on the structural characteristics of the produced intermediate colloidal phases, media containing LPC instead of PL with (free fatty acid) FFA/MG ratios of 2:1 and 6:1 were prepared. In addition, Chol was added in a ratio of FFA/Chol 20:1 corresponding to the approximate ratio found in human intestinal fluids (6).

\section{MATERIALS AND METHODS \\ Materials}

Oleic acid, sodium taurocholate, cholesterol, sodium chloride, sodium azide, lyso-phosphatidylcholine, and trizma maleate were purchased from Sigma (St. Louis, MO). Danisco (Denmark) generously donated glycerol monoolate (Monoolein). Water was obtained from a MilliQ water purification system manufactured by Millipore (USA.) All other chemicals were of analytical grade.

\section{Preparation of Biorelevant Media}

The composition of the biorelevant media is presented in Table 1. Combinations of sodium taurocholate (bile salt, $\mathrm{ST})$, lecithin (phospholipid, PC), monoolein (monoglyceride, MO), and oleic acid (fatty acid, OA) were prepared at different ratios. The biorelevant media were prepared by 
Table 1. Composition of Biorelevant Media (mM)

\begin{tabular}{llllll}
\hline Medium & LPC & ST & OA & MO & Chol \\
\hline I & 3.75 & 15 & 15 & 7.5 & 0.75 \\
\hline II & 3.75 & 15 & 15 & 2.5 & - \\
\hline III & 3.75 & 15 & 15 & 2.5 & 0.75 \\
\hline IV & 5 & 20 & 20 & 3.33 & 1 \\
\hline
\end{tabular}

LPC: Lyso-phosphatidylcholine, ST: Sodium Taurocholate, OA: Oleic Acid, MO: Monoolein, Chol: Cholesterol.

weighing the exact amounts of the components. The osmolarity of the buffer solution was fixed at $270 \mathrm{mOsm} /$ $\mathrm{kg}$, included $100 \mathrm{mM}$ trizma maleate at $\mathrm{pH} 6.5$, and contained $65.1 \mathrm{mM} \mathrm{NaCl}$. Buffer solution was added to give the desired molar ratios and left for $24 \mathrm{~h}$ under stirring at $37^{\circ} \mathrm{C}$. To prevent microbial growth, $3 \mathrm{mmol}$ of sodium azide was added. Each medium was prepared in triplicate.

\section{Size and $\zeta$-Potential Studies}

Biorelevant media were measured at $25^{\circ} \mathrm{C}$ using dynamic light scattering (DLS) (Malvern Nanosizer ZS, Malvern Instruments, UK). The refractive index and viscosity of the water used in the study were 1.333 and $0.891 \mathrm{cP}$, respectively. The $\zeta$-potential of the dispersions was calculated by the instrument according to the Helmholtz-Smoluchowski equation. Both size and $\zeta$-potential determinations were performed in triplicate.

\section{Cryo-Transmission Electron Microscopy (Cryo-TEM)}

The results presented here are the outcomes for two different batches of each medium and an evaluation of 89 images.

The samples for the Cryo-TEM studies were prepared in a controlled-environment vitrification system (CEVS). A small amount of the sample $(5 \mu \mathrm{L})$ was put on a carbon film supported by a copper grid and blotted with filter paper to obtain a thin liquid film on the grid. The grid was quenched in liquid ethane at $-180^{\circ} \mathrm{C}$ and transferred to liquid nitrogen $\left(-196^{\circ} \mathrm{C}\right)$. The samples were characterized with a transmission electron microscope (Philips CM120 BioTWIN Cryo) equipped with a post-column energy filter (GATAN GIF 100) using an Oxford CT 3500 cryoholder and workstation. The acceleration voltage was $120 \mathrm{kV}$ and the working temperature was $-180^{\circ} \mathrm{C}$. The images were recorded with a CCD camera (Gatan 791) under low dose conditions. The defocus was approximately $1 \mu \mathrm{m}$.

\section{RESULTS AND DISCUSSION}

\section{Size and $\zeta$-Potential Studies}

The relationship between the total concentration of anionic species and their $\zeta$-potential values is illustrated in Figure 1. Overall, changes to the $\zeta$-potential of the media are determined by the relative contribution of each component separately. BS and fatty acids (FA) possess a

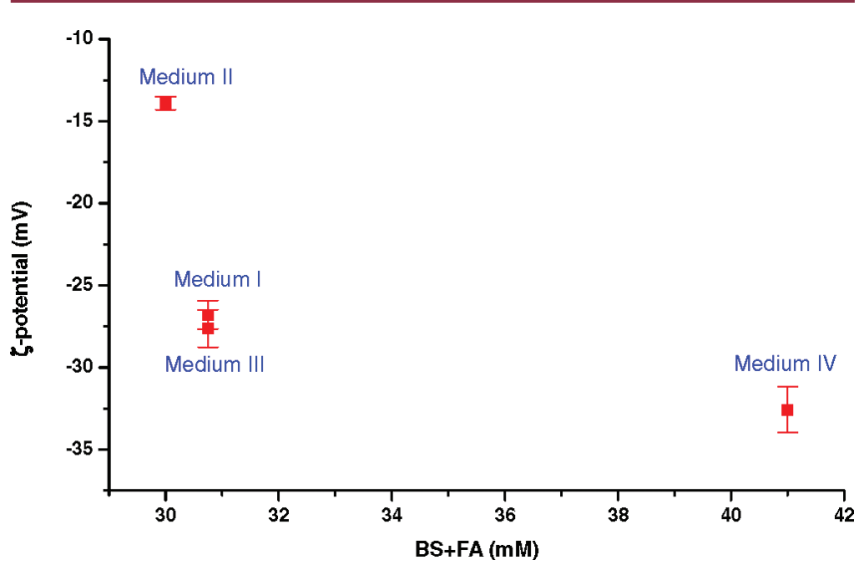

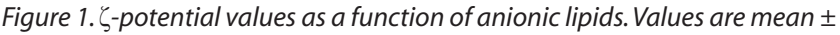
SD (standard deviation) of three experiments.

negative charge, while LPC and Chol are zwitterionic with no net charge. On the other hand, monoolein is a neutral lipid and does not contribute to the $\zeta$-potential. Medium IV exhibits the highest $\zeta$-potential values in absolute numbers $(-32.56 \mathrm{mV})$ due to higher levels of oleic acid $(\mathrm{OA})$ and MO. The differences in the $\zeta$-potential values between medium II and media I, III, and IV can be attributed to the presence of cholesterol in the latter. Previous studies (11) have shown that the $\zeta$-potential of cholesterol in an aqueous medium is positive $(\mathrm{pH} 1)$, reaching a zero value when $\mathrm{pH} \approx 2.9$, which is the isoelectric point, and decreasing to negative values with a further increase in $\mathrm{pH}$. These results can be qualitatively explained by assuming that an increasing $\mathrm{OH}^{-}$adsorption is taking place on the cholesterol surface, increasing the negative charge of the media.

The dominant particles for media II, III, and IV were micelles with a particle size range of 5.69-8.05 nm (Table 2). In medium I, larger particles were also present; this heterogeneity rendered it impossible to measure the particle size distribution with DLS.

\section{Cryo-TEM Studies}

Figure 2 depicts the Cryo-TEM images of colloidal structures present in medium I. Vesicle diameters from 50 to $800 \mathrm{~nm}$ were seen. Undulations and ripples were visualized on the bilayers in the inner part of the vesicles (Figure 2C, black arrow). Occasionally, ruptured vesicles were recognized as well (Figure 2D, white arrow). The main components in these colloidal phases were MO, OA, and PC. These colloidal phases closely resembled PL liposomes in their structure and morphological properties. Vesicles with a deformed internal structure were recognized, suggesting surface tension or uneven lateral stress of these particles (Figure 2C). Most probably, these are intermediate structural features that have not yet reached equilibrium.

Cryo-TEM images of media II, III, and IV are depicted in Figure 3. In all cases, the dominating structures are micelles, seen as black "dots", containing ST and LPC in 
Table 2. Size Distribution of Structures or Micelles in Simulated Intestinal Fed-State Fluids ( $\mathrm{nm}$ )

\begin{tabular}{lcl}
\hline Medium & Size & $\begin{array}{c}\text { Formed Structures in } \\
\text { the Medium }\end{array}$ \\
\hline I & Not measured & Micelles and Vesicles \\
\hline II & $5.69 \pm 0.56$ & Micelles \\
\hline III & $8.05 \pm 1.98$ & Micelles \\
\hline IV & $6.75 \pm 0.26$ & Micelles \\
\hline
\end{tabular}
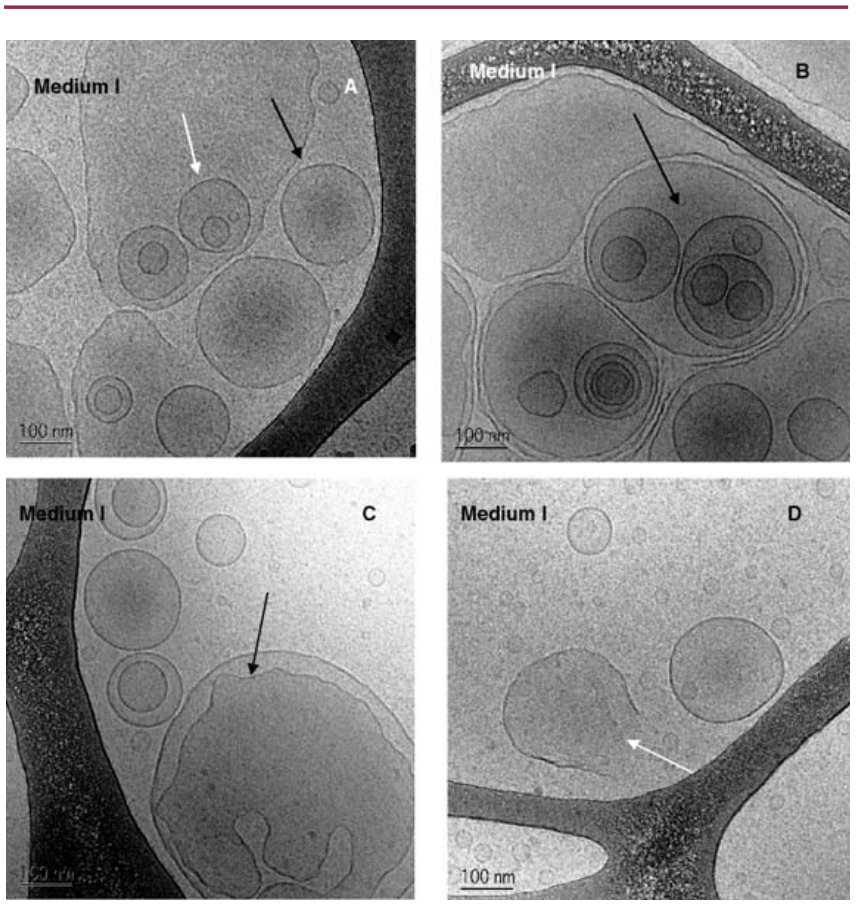

Figure 2. Cryo-TEM images of simulated intestinal fluids (medium I). Bar represents $100 \mathrm{~nm}$. (A) Unilamellar (black arrow), bi-lamellar (white arrow), (B) multi-compartmental vesicles (black arrow) co-existing with (A) micelles (black dots) were present. (C) Undulations and ripples (black arrow). (D) Ruptured vesicles (white arrow).

levels representative of the fed state. The data obtained from the Cryo-TEM studies for media II, III, and IV are in good agreement with the DLS studies.

To summarize, the following observations have been made regarding the colloidal structures in the tested media:

1) The replacement of $P C$, an amphiphile known to induce lamellar and vesicular phases (12), with LPC, which forms micelles when dispersed in aqueous enviroments (13), in the media containing OA/MO 6:1 (media II, III, and IV) eliminated the formation of vesicles. Micelles were the dominating structural features with particle sizes between 5.6 and $8.0 \mathrm{~nm}$.

2) Media I and III are identical except for an additional 5 $\mathrm{mM}$ of $\mathrm{MO}$ in medium I. This leads to the formation of vesicles that coexist with micelles in the medium. This
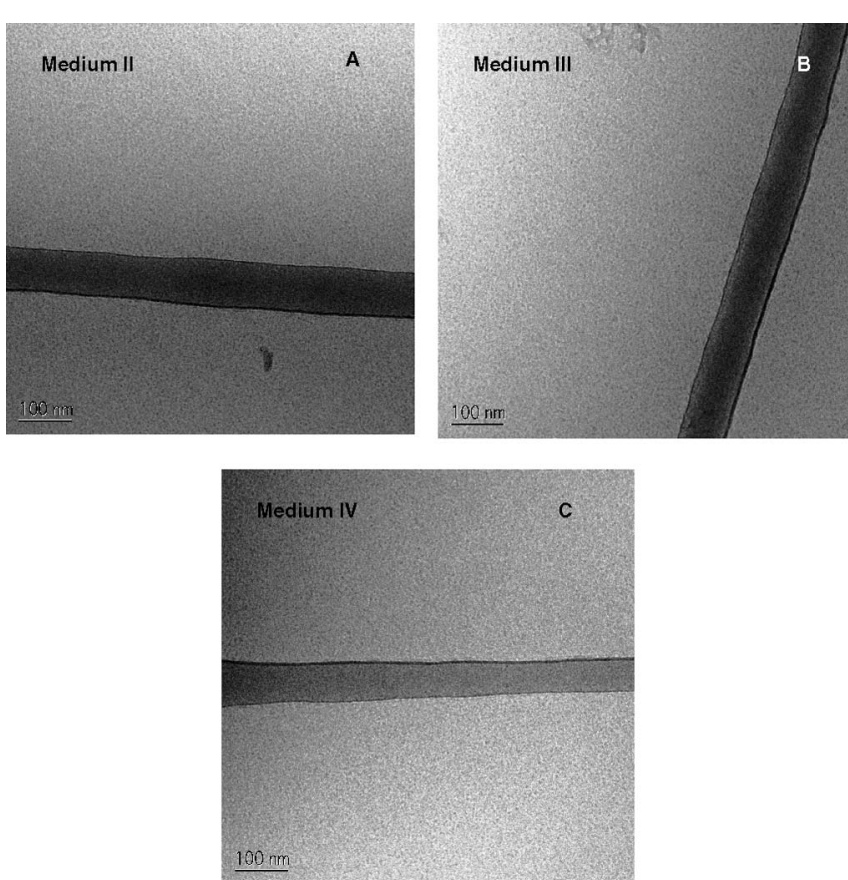

Figure 3. Cryo-TEM images of simulated intestinal fluids (media II, III, and IV). Bar represents $100 \mathrm{~nm}$.

can be explained by the swelling characteristics of $\mathrm{MO}$ and its ability to form lyotropic liquid crystals (14).

3) The presence of low levels of cholesterol increases the absolute $\zeta$-potential values of the media (compare medium II with I and III); however, it has little or no effect on the colloid structures present in the media.

There was no evidence of cholesterol monohydrate crystals in the media. Such structural features have been visualized by Cryo-TEM previously in human bile where cholesterol concentrations were between 5 and $18 \mathrm{mM}$ (15). The structures observed in the current study are in broad agreement with previous reports where model lipid systems containing monoglycerides, oleic acid, and sodium taurodeoxycholic acid were visualized by freezefracture electron microscopy (12). In samples with high concentrations of lipolytic products, unilamellar and multilamellar vesicles were visualized. Additionally, long chains FFA have demonstrated their ability to form unilamellar vesicles upon dispersion in aqueous solutions (16). Figure 4 illustrates typical structures seen in a previous study (17) with media containing the same ratios of ST, OA, and PC (instead of LPC), as the in the present study, but with higher amounts of MO.

\section{CONCLUSION}

The solubility profiles of APIs are greatly affected by the diversity and complexity of the media in terms of structure and size, them emphasizing the important interaction between a lipid-based formulation and the endogenous lipid system. Therefore, it is important to gain a better understanding of the impact of these species on the intermediate colloid phases present in the Gl tract during 

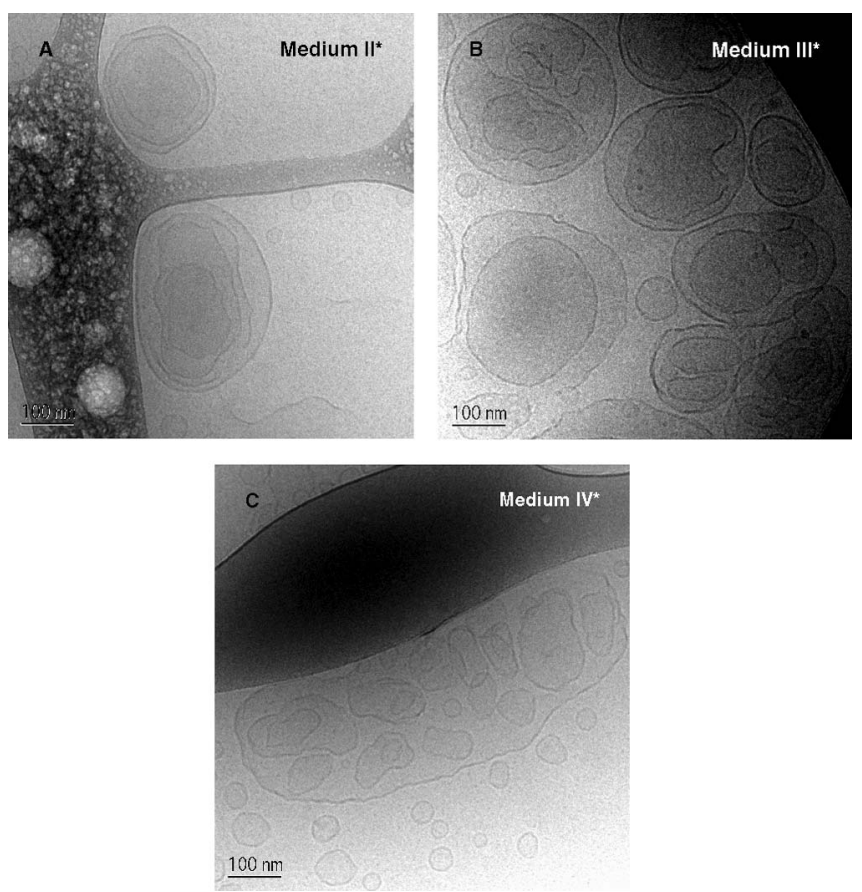

Figure 4. Cryo-TEM images of simulated intestinal fluids containing PC instead of $L P C$ and high ratios of $M G$. In all cases, $(A)$ multilamellar, $(B)$ internal deformed structures, and (C) multivesicular structures were identified to the presence of PC and MG in broad agreement with the structures recognized in Medium I. (Reprinted with permission from ref 17. Copyright 2009 Springer.)

drug dissolution. This work sheds some light on these structures and lays the framework for future studies.

\section{ACKNOWLEDGMENTS}

The Cryo-microscopy was performed at the Biomicroscopy unit of the Centre of Chemistry and Chemical Engineering at Lund University. The authors are grateful to Mrs. Gunnel Karlsson for her skillful assistance with the Cryo-TEM instrument. This work was financially supported by the Drug Research Academy (DRA), the Danish University of Pharmaceutical Sciences.

\section{REFERENCES}

1. Bates, T. R.; Lin, S. L.; Gibaldi, M. Solubilization and rate of dissolution of drugs in the presence of physiologic concentrations of lysolecithin. J. Pharm. Sci. 1967, 56, 1492-1495.

2. Serajuddin, A.T.; Sheen, P.C.; Mufson, D.; Bernstein, D. F.; Augustine, M. A. Physicochemical basis of increased bioavailability of a poorly water-soluble drug following oral administration as organic solutions. J. Pharm. Sci. 1988, 77, 325-329.

3. Armand, M.; Borel, P.; Pasquier, B.; Dubois, C.; Senft, M.; Andre, M.; Peyrot, J.; Salducci, J.; Lairon, D. Physicochemical characteristics of emulsions during fat digestion in human stomach and duodenum. Am.J. Physiol. 1996, 271, G172-G183.
4. Tangerman, A.; van Schaik, A.; van der Hoek, E. W. Analysis of conjugated and unconjugated bile acids in serum and jejunal fluid of normal subjects. Clin. Chim. Acta 1986, 159, 123-132.

5. Galia, E.; Nicolaides, E.; Hörter, D.; Löbenberg, R.; Reppas, C.; Dressman, J. B. Evaluation of Various Dissolution Media for Predicting In Vivo Performance of Class I and II Drugs. Pharm. Res. 1998, 15, 698-705.

6. Persson, E. M.; Gustafsson, A. S.; Carlsson, A. S.; Nilsson, R. G.; Knutson, L.; Forsell, P.; Hanisch, G.; Lennernas, H.; Abrahamsson, B. The effects of food on the dissolution of poorly soluble drugs in human and in model small intestinal fluids. Pharm. Res. 2005, 12, 2141-2151.

7. Jantratid, J.; Janssen, N.; Reppas, C.; Dresmman, J. B. Dissolution media simulating conditions in the proximal human gastrointestinal tract:an update. Pharm. Res. 2008, 25, 1663-1676.

8. Clarysse, S.; Tack, J.; Lammert, F.; Duchateau, G.; Reppas, C.; Augustijns, P. Postprandial evolution in composition and characteristics of human duodenal fluids in different nutritional states. J. Pharm. Sci. 2009, 98, 1177-1192.

9. Kossena, G. A.; Boyd, B. J.; Porter, C. J.H.; Charman, W. N. Separation and characterization of the colloidal phases produced on digestion of common formulation lipids and assessment of their impact on the apparent solubility of selected poorly water-soluble drugs. J. Pharm. Sci. 2003, 92, 634-648.

10. Kossena, G. A.; Charman, W. N.; Boyd, B. J.; Porter, C. J.H. Influence of the intermediate digestion phases of common formulation lipids on the absorption of a poorly water-soluble drug.J.Pharm. Sci. 2005, 94, 481-492.

11. Salcedo, J.; Delgado, A.; Gonzalez-Cabalero, F. The effect of $\mathrm{pH}$ on the electrical properties of the cholesterol/water interface. I. $\zeta$-potential. J. Coll. Inter. Sci. 1989, 133, 278-281.

12. Rigler, M.W.; Honkanen, R. E.; Patton, J. S. Visualization by freeze fracture, in vitro and in vivo, of the products of fat digestion. J. Lipid Res. 1986, 27, 836-857.

13. Saunders, L.; Thomas, I. L. Diffusion studies with lysolecithin. J. Chem. Soc. 1958, 483-485.

14. Caffrey, M. The study of lipid phase transition kinetics by time-resolved X-ray diffraction. Ann. Rev. Biophys. Biochem. Chem. 1989, 18, 159-186.

15. Konikoff, F. M.; Danino, D.; Weihs, D.; Rubin, M.; Talmon, Y. Microstructural evolution of lipid aggregates in nucleating model and human biles visualized by cryogenic transmission electron microscopy. Hepatology 2000, 31, 261-268.

16. Hargreaves, W. R.; Deamer, D.W. Liposomes from ionic, single-chain amphiphiles. Biochemistry 1978, 17, 3759-3768.

17. Fatouros, D. G.; Walrand, I.; Bergenstahl, B.; Müllertz, A. Colloidal structures in media simulating intestinal fed-state conditions with and without lipolysis products. Pharm. Res. 2009, 26, 361-374. 